\title{
RELATIVISTIC QUANTUM CHAOS IN \\ DE SITTER COSMOLOGIES
}

\author{
Roman Tomaschitz \\ Dipartimento di Matematica Pura ed Applicata dell' Università degli Studi di Padova, \\ Via Belzoni 7, I-35131 Padova, Italy \\ and
}

Dipartimento di Fisica dell' Università di Bologna, Via Irnerio 46, I-40126 Bologna, Italy

and

Service de Physique Théorique de Saclay, F-91191 Gif -sur-Yvette, France

\begin{abstract}
We consider classical and quantal motion in open de Sitter cosmologies of multiple spatial connectivity. The topological structure of the spacelike slices creates on the one hand chaotic trajectories, on the other hand bound states whose wave fields and energies are intimately connected with the Hausdorff dimension and measure of limit sets of chaotic trajectories in the covering space of the manifold. We discuss the time evolution of of the energy of wave fields coupled to the curvature scalar of the 4-manifold in the early and late stages of the cosmic expansion, and its dependence on the spectral variable of the Laplace-Beltrami operator on the space sections. This spectrum is in turn entirely determined by the topological and metrical structure of the sections.
\end{abstract}

\section{Introduction}

In this paper we will address questions concerning the influence of the global topological structure of space-time on the microscopic laws of motion. At fist, in how far does the topology of the spacelike sections of Robertson-Walker Cosmologies influence the time evolution of the energy of wave fields, the quantum version of geodesic motion on world lines. Secondly we discuss the reconstruction of quantal wave fields from chaotic classical world lines. These world lines having the Bernoulli property are a typical consequence of the multiple connectivity of 3-space, and are foreign to simply connected spaces, traditionally encountered in cosmological models. 
The first question is in some sense paraphrasing Mach's principle, namely that the global structure of the universe determines the local laws of of motion, inertial forces being gravitational, stemming from the mass content of the whole (closed) universe. Our emphasis lies more on the topological structure of the open universe, which is of course in turn related with the energy momentum tensor of the mass distribution.

In the second problem it might seem strange that it is possible to reconstruct a non-deterministic wave field from classical geodesics. The point is here that we actually relate two probabilistic models of microscopic motion, that of quantum mechanics and that of unstable classical dynamics, the classical trajectories in question having positive Lyapounov exponents.

\section{Open Robertson-Walker cosmologies of multiple connectivity}

We consider cosmological line elements of the form

$$
d s^{2}=-c^{2} d t^{2}+a^{2}(t) d \sigma^{2},
$$

where $d \sigma^{2}$ is the metric of hyperbolic 3-space, which we may represent in the coordinates of the Poincaré ball $\mathrm{B}^{3},|\overrightarrow{\mathbf{x}}|<\mathrm{R}$ (cf.[1]),

$$
d \sigma^{2}=\frac{4 d \vec{x}^{2}}{\left(1-|\vec{x}|^{2} / R^{2}\right)^{2}}
$$

The expansion factor of 3-space a(t) determines the Gaussian curvature $\mathrm{K}=-1 /(\mathrm{aR})^{2}$ of the spacelike sections $\mathrm{t}=$ const. .The spacelike projections of the geodesics into $B^{3}$ calculated via (2.1) are arcs of semicircles orthogonal to $S_{\infty}$, the sphere at infinity (boundary) of $\mathrm{B}^{3}$, where the Poincaré metric gets singular. The totally geodesic planes in $\mathrm{B}^{3}$ are thus spherical caps orthogonal to $S_{\infty}$.

Hyperbolic manifolds, i.e. manifolds of constant negative curvature, are modelled in $\mathrm{B}^{3}$ as non-Euclidean polyhedra, whose faces lie on geodesic planes. The faces are identified in pairs by elements of the Lorentz group, acting in $\mathrm{B}^{3}$ as invariance group of (2.2), defining in this way the topology ( compare the well known example of a torus represented in the Euclidean plane as a square with identified sides). The polyhedron may have 'free' faces on $S_{\infty}$, which are not identified in pairs and which constitute the boundary at infinity of the manifold. The face-pairing transformations generate a discrete subgroup $\Gamma$ of the Lorentz group, the group of covering transformations of the manifold, and $B^{3}$ is its universal covering space. The set $\Gamma(F)$ of all images of $F$ under $\Gamma$ gives a cover (tiling) of $B^{3} . \Gamma(F)$ as an infinite number of tiles in $B^{3}$ has accumulation points, 
the limit set $\Lambda(\Gamma)$ of $\Gamma$. For the manifolds that we consider here, which have the topology I $\times S, I$ an open finite interval, $S$ a compact Riemann surface, $\Lambda(\Gamma)$ is a Jordan curve on $S_{\infty}$. Figs. $(1,2)$ show these limit sets and the interior part of the tiling induced on $S_{\infty}$ from the covering of the ball $\mathrm{B}^{3}$. The tiles are images of one of the boundary surfaces (free faces) of the 3-manifold under the covering group $\Gamma$ on $S_{\infty}$.

The topological structure of 4-space is now $R^{+} \times I \times S, R^{+}$the time axis $0<t<\infty$, and $I \times S$ is represented in $B^{3}$ as a polyhedron $F$ with face-identification by $\Gamma$. The metric of $B^{3}$ is induced on $F$. We mention that $F$ and $\Gamma$ could also vary in time, constituting a one-parameter family $(F(t), \Gamma(t))$ of non-isometric 3-manifolds. Even the topological structure of 3-space may change, e.g. it could disintegrate by developing parabolic cusp singularities at the boundary of the deformation space ( $\mathrm{cf} .[2,3])$.

We call a trajectory bounded if it stays in a finite region that is not expanding faster then 3-space itself. The bounded chaotic trajectories are intimately connected with the limit set $\Lambda(\Gamma)$ (cf.[7]). For example if the expansion factor $a(t)$ is of the order $t^{\alpha}$, $\alpha \geq 1$ for $t \rightarrow 0$, a trajectory is only then bounded for $t \rightarrow 0$, if its lifts in the covering space emanate from $\Lambda(\Gamma)$, and has then the Bernoulli property. Likewise, if $a(t)$ is of order $t^{\beta}, \quad \beta \leq 1 / 2$, for $t \rightarrow \infty$, it is only then bounded for $t \rightarrow \infty$ if its lifts end in the limit set $\Lambda(\Gamma)$. Thus in the case of de Sitter space, $a(t)=\sinh (\Lambda t)$, the bounded trajectories are exactly those that emanate from $\Lambda(\Gamma)$ (cf.[8]).

The energy-momentum tensor $\hat{\mathrm{T}}_{\mu \nu}$ produced by the metric $\mathrm{g}_{\mu v}$ in $(2.1)$ we obtain via the Einstein tensor : $8 \pi \mathrm{k} / \mathrm{c}^{4} \widehat{\mathrm{T}}_{\mu v}:=\widehat{R}_{\mu \nu}-1 / 2 \mathrm{~g}_{\mu v} \widehat{R}+\lambda \mathrm{g}_{\mu v}$, with $\hat{R}_{00}=-3 a / a, \hat{R}_{i j}=4\left(1-|\vec{x}|^{2} / R^{2}\right)^{-2} \delta_{i j}\left[c^{-2}\left(a a+2 \mathfrak{a}^{2}\right)-2 / R^{2}\right], \hat{R}_{0 j}=0$ (sign conventions as in [5] ). We have to show that $\hat{\mathrm{T}}_{\mu v}$ can be brought into the form $\hat{\mathrm{T}}_{\mu \nu}=(\varepsilon+p) \mathrm{u}_{\mu} \mathrm{u}_{v}+\mathrm{pg}_{\mu \nu}$, with $\varepsilon, \mathrm{p}, \mathrm{u}_{\mathrm{k}}$ automorphic functions of $\Gamma$ (periodic with respect to $\Gamma$ ) in $\mathrm{B}^{3}$, so that their restriction onto $\mathrm{F}$ gives smooth fields on the manifold, in particular on the identified polyhedral faces ${ }^{*}$. We choose as in the topologically trivial case $u=(c, \overrightarrow{0})$ (comoving frame); $\varepsilon$ and $p$ are then functions of $a(t)$ and its first and second derivatives, and $\mathrm{g}_{\mu \nu}$ is automorphic with respect to any discrete subgroup of the Lorentz group. It would be interesting to see if this holds true if we relax the condition of constant curvature of 3-space and allow local perturbations of the metric (see also Section IV).

\footnotetext{
* I would like to thank N. Balazs for pointing out this consistency check.
} 


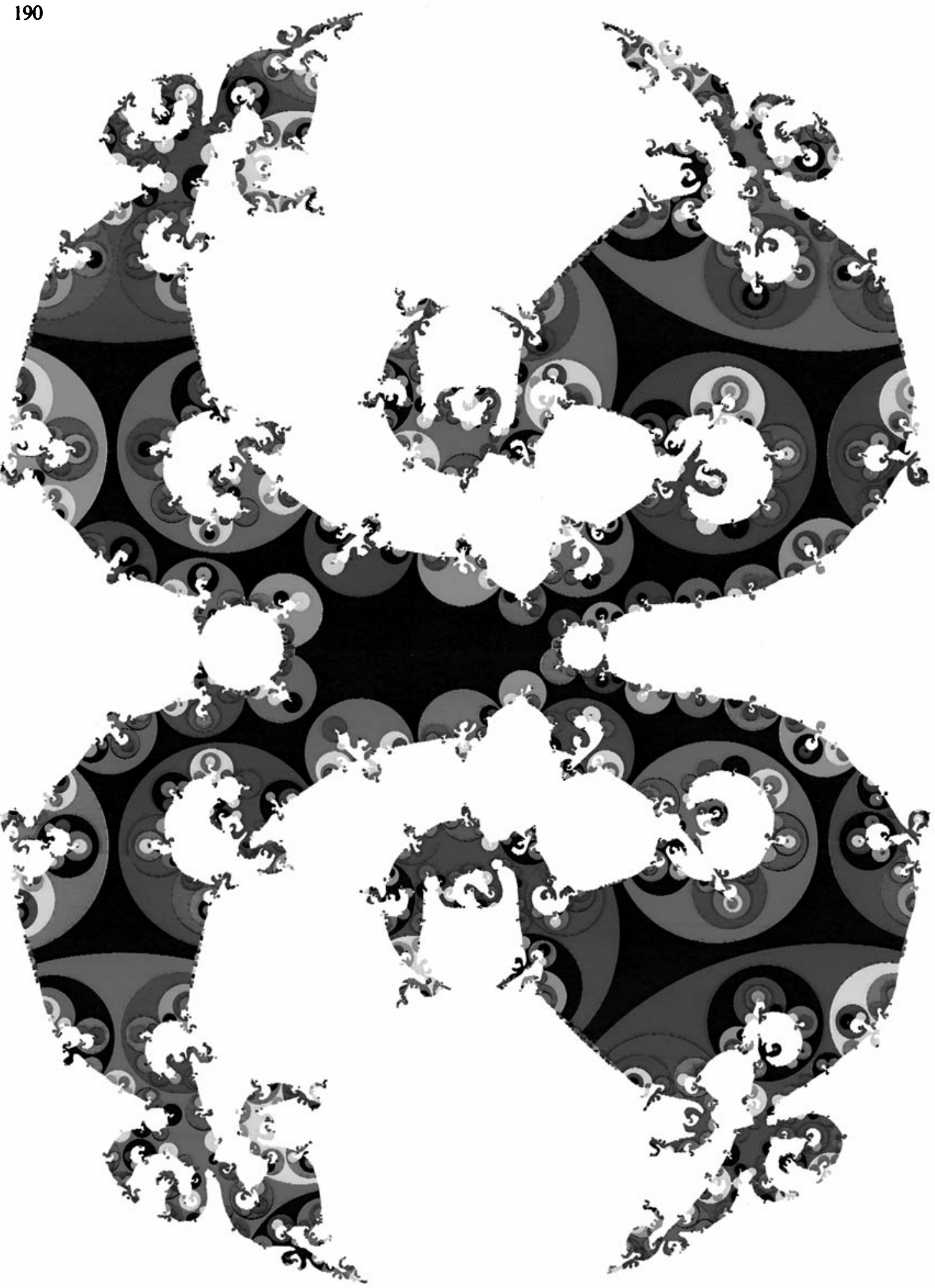

Fig. 1 : Tiling induced on the sphere at infinity of the Poincare ball by the universal cover of the manifold. The quasi self-similar Jordan curve determines the bounded trajectories, and its Hausdorff measure the corresponding chaotic wave fields. $g(S)=19, \delta=1.382$. 


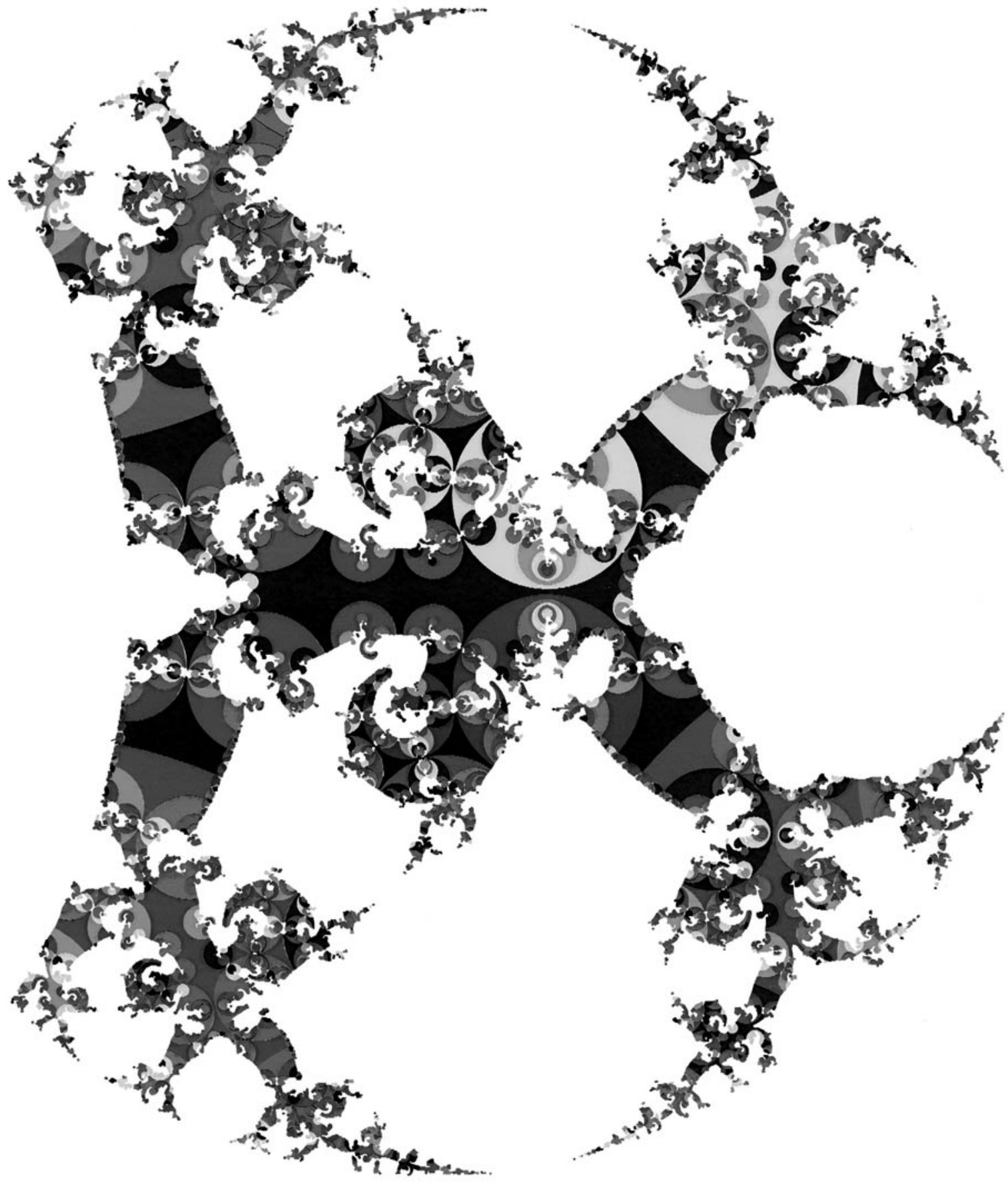

Fig. 2 : As Fig. 1, covering of $S_{\infty}$ stemming from a section $t=$ const. of the 4-manifold. Figs. 1, 2 show tilings of two non-isometric points on a path $(F(t), \Gamma(t))$ in the deformation space of the topological manifold $I \times S . g(S)=19, \delta=1.424$. 


\section{Wave mechanics}

The wave equation on the covering space $R^{+} \times B^{3}$ reads

$$
\left[\square-\xi \widehat{R}-(\mathrm{mc} / \mathrm{h})^{2}\right] \psi=0,
$$

is the Laplace-Beltrami operator of the line element $\mathrm{ds}^{2}$ in $\mathrm{R}^{+} \times \mathrm{B}^{3}$, and $\xi$ the (dimensionless) coupling to the curvature scalar $\hat{\mathbf{R}}$.

Separation of variables $\psi(t, \vec{x})=\hat{\psi}(\vec{x}) \varphi(t)$ in (3.1) gives

$$
\left[\Delta_{B^{3}}+R^{-2} \delta(2-\delta)\right] \hat{\psi}=0
$$

$\Delta_{\mathrm{B}}{ }^{3}$ is the L.-B.-operator of $\mathrm{B}^{3}$ endowed with the Poincare metric (2.2) and $\mathrm{R}^{-2} \delta(2-\delta)$, the separation constant, appears as spectral parameter. We keep here $(F, \Gamma)$ independent of time, otherwise eq.(3.1) is not separable. For $\varphi$ we have

$$
\ddot{\varphi}+3 \frac{\hat{\mathrm{a}}(\mathrm{t})}{\mathrm{a}(\mathrm{t})} \dot{\varphi}+\left[\left(m \mathrm{c}^{2} / \mathrm{n}\right)^{2}+\Lambda^{2} \delta(2-\delta) \mathrm{a}^{-2}(\mathrm{t})+\mathrm{c}^{2} \xi \hat{\mathrm{R}}(\mathrm{t})\right] \varphi=0
$$

with $\Lambda=c / R$.

The wave equation on the manifold $\mathrm{R}^{+} \times \mathrm{I} \times \mathrm{S}, \mathrm{I} \times \mathrm{S}$ represented as the polyhedron $F$ in $B^{3}$ is just eq. (3.1) subject to periodic boundary conditions on the identified faces of the polyhedron, which amounts to require periodicity of $\psi$ in $\mathrm{B}^{3}$ with respect to the group $\Gamma, \psi(t, \gamma \overrightarrow{\mathrm{x}})=\psi(\mathrm{t}, \overrightarrow{\mathrm{x}})$ for all $\gamma$ in $\Gamma$.

Concerning the time dependence of $\psi$ we impose the following end-value condition on $\varphi(t)$ (positive frequency solutions): $\varphi(t) \sim f(t) e^{-i g(t)}$ for $t \rightarrow \infty$, with real $f, g ; f(t)$ monotonically decreasing, and $g(t)$ strictly monotonically increasing, thus approximating as well as possible the Minkowski space solutions in the asymptotic regime, when 3-space gets flat .

About the spectrum of ( 3.2 ) the following is known [6]. There is a unique discrete eigenvalue for a $\delta$ in the interval $[1,2)$. This $\delta$ is just the Hausdorff dimension of $\Lambda(\Gamma)$. We have absolutely continuous spectrum for $\delta(2-\delta)>1$. The square-integrable 
wave function on $\mathrm{F}$ belonging to the discrete eigenvalue has an integral representation with a Poisson kernel and the Hausdorff measure $d \mu$ of $\Lambda(\Gamma)$,

$$
\hat{\psi}(\vec{x})=\int_{\Lambda(n)} \frac{\left(1-|\vec{x}|^{2} / R^{2}\right)^{\delta}}{|\vec{x}-\vec{\eta}|^{2 \delta}} d \mu(\vec{\eta})
$$

The eigenfunctions of the continuous spectrum are Eisenstein series $[3,6]$.

With the covariant and indefinit scalar product for wave fields the normalization condition for $\varphi$ reads [8]

$$
\frac{1}{2}(\dot{\varphi} \varphi-\dot{\varphi} \bar{\varphi})= \pm \mathrm{i} \mathrm{a}^{-3}(\mathrm{t})
$$

where $\varphi, \bar{\varphi}$ is a pair of fundamental solutions of (3.3).

The energy -momentum tensor $T_{\mu \nu}$ of the field in (3.1) we get by variation of the action functional with respect to the metric, cf.[4]. The energy of the wave field is calculated as

$$
\mathrm{E}=\pi \mathrm{c}^{2} \int_{\Sigma} \mathrm{T}_{0 \mu} \mathrm{d} \Sigma^{\mu}
$$

with $\Sigma$ an arbitrary spacelike hypersurface.

We have then

$$
E(t, \delta)=\frac{1}{2} \hbar a^{3}\left\{\left(\varphi, c+6 \xi \varphi \frac{\hat{a}}{a}\right) \overline{\left(\varphi, t+6 \xi \varphi \frac{\hat{a}}{a}\right)}+\varphi \bar{\varphi}\left[\left(\frac{m c^{2}}{\hbar}\right)^{2}+\Lambda^{2}[\delta(2-\delta)-6 \xi] a^{-2}+6 \xi(1-6 \xi) \frac{\hat{a}^{2}}{a^{2}}\right]\right\} .
$$

This expression is obviously positive definit for $0 \leq 6 \xi \leq \min [1, \delta(2-\delta)]$, but actually positivity is too restrictive a condition, for example in the treatment of massless particles. What we will require is uniform boundedness of the energy functional from below. 
We discuss ( 3.6 ) valid for every Robertson - Walker line element in the context of de Sitter space. We have $a(t)=\sinh (\Lambda t), 3 \Lambda^{2} / c^{2}$ is the cosmological constant (denoted by $\lambda$ in Section II), $\hat{R}=12 \Lambda^{2} / c^{2}$, and $R=c / \Lambda$. The general solution of (3.3) is in this case in terms of hypergeometric functions [8]. We define

$$
\kappa^{2}=-1 / 4-2(1-6 \xi)+\left(m c^{2} / \mathrm{h \Lambda}\right)^{2} \text {, and } v^{2}=1-\delta(2-\delta) \text {, }
$$

and assume $k^{2}>0$ to ensure the right time behaviour at infinity. The positive frequency solution for $t \rightarrow \infty$ is then

$$
\varphi(t)=(\Lambda|\kappa|)^{-1 / 2} 2^{(3 / 2+i \kappa)} e^{-\Lambda t(3 / 2+i k)}\left\{1+\frac{e^{-2 \Lambda t}}{1+i \kappa}\left[\frac{5}{4}+v^{2}+\frac{3}{2} i \kappa\right]+O\left(e^{-4 \Lambda t}\right)\right\},
$$

and for $t \rightarrow 0, v>0$ (discrete spectrum),

$$
\varphi_{g . s t .}(t) \sim \frac{2^{i k}}{\sqrt{2 \pi|k| \Lambda}} \frac{\Gamma(1+i k) \Gamma(v) 2^{v}}{\Gamma(1 / 2+i \kappa+v)}(\Lambda t)^{-v-1} .
$$

For $t \rightarrow 0, \operatorname{Im}(v)>0$ (continuous spectrum),

$$
\varphi(t) \sim \frac{2^{i \kappa} \Gamma(1+i \kappa)}{\sqrt{2 \pi|\kappa| \Lambda}}\left[\frac{\Gamma(-v) 2^{-v}}{\Gamma(1 / 2+i \kappa-v)}(\Lambda t)^{v-1}+\frac{\Gamma(v) 2^{v}}{\Gamma(1 / 2+i \kappa+v)}(\Lambda t)^{-v-1}\right] .
$$

The negative frequency solutions are conjugated to them.

With these solutions we obtain the time evolution of energy in the asymptotically flat region,

$$
E(t \rightarrow \infty, m>0) \sim \frac{m^{2} c^{4}}{\hbar \Lambda|k|}=m c^{2}+O\left(\hbar^{2}\right),
$$

and

$$
\mathrm{E}(\mathrm{t} \rightarrow \infty, \mathrm{m}=0) \sim \frac{2 \hbar \Lambda(1-6 \xi)[36 \xi-5 \delta(2-\delta)]}{\sqrt{12 \xi-9 / 4}(12 \xi-5 / 4)} \mathrm{e}^{-2 \Lambda t} .
$$

The leading order of the asymptotic expansion is independent of the spectral variable $v$. 
At the beginning of the evolution, for $t \rightarrow 0$, we have

$$
E(v>0, t \rightarrow 0) \sim \frac{\hbar \Lambda 2^{2 v-1} \Gamma^{2}(v)(1+v)(1-6 \xi)}{\sinh (\pi|\kappa|)|\Gamma(1 / 2+v+i k)|^{2}}(\Lambda t)^{-2 v-1}
$$

Finally, for $\operatorname{Im}(v)>0$, writing $v=i \tilde{v}, \tilde{v}=\sqrt{\delta(2-\delta)-1}>0$,

$$
\mathrm{E}(\operatorname{Im}(v)=\tilde{v}>0, t \rightarrow 0) \sim \frac{\hbar}{t}\left\{A(\tilde{v}, \kappa, \xi)+\operatorname{Re}\left[B(\tilde{v}, \kappa, \xi)(\Lambda t)^{2 i \tilde{v}}\right]\right\},
$$

with

$$
\mathrm{A}(\tilde{v}, \kappa, \xi)=\tilde{v}^{-1} \operatorname{coth}(\pi|\kappa|) \operatorname{coth}(\pi \tilde{v})\left(\tilde{v}^{2}+1-6 \xi\right),
$$

and

$$
\mathrm{B}(\tilde{v}, \kappa, \xi)=\frac{(1-6 \xi)(1-\tilde{i})}{\sinh (\pi|\kappa|)} \frac{\Gamma^{2}(-i \tilde{v}) 2^{-2 i \tilde{v}}}{\Gamma(1 / 2-i \tilde{v}+i \kappa) \Gamma(1 / 2-i \tilde{v}-i \kappa)}
$$

In the initial state of the cosmic expansion, for $t \rightarrow 0$, there is an infinite oscillation of $E$ ( eq. (3.14)) between the two curves ${ }^{\hbar t}{ }^{-1}(A \pm|B|)$, with an amplitude $\hbar t^{-1}|B|$, and a frequency $\left(e^{\pi \tilde{N}}-1\right)^{-1} t^{-1}$, both diverging to infinity $(A-|B|>0$, see below). On the other hand, the energy associated with the discrete eigenvalue (eq. (3.13), and $1-6 \xi>0$ ) does not fluctuate at all, and overpowers that of the continuous spectrum eq. ( 3.14 ) for any fixed $\tilde{v}$. However, we see from the following asymptotic expansion,

$$
\mathrm{E}(\tilde{v} \rightarrow \infty, t)=\frac{\hbar \Lambda}{a(t)} \tilde{v}\left[1+O\left(1 \tilde{v}^{2}\right)\right],
$$

valid for any fixed $t$, that it lies well inside the range of energies obtainable from the continuous spectrum. We compare these formulae with the classical energy, $\mathrm{E}=\mathrm{mc}^{2} \sqrt{1+\text { const. }^{-2}(\mathrm{t})}$. The constant depends on the initial velocity, and $\mathrm{E}$ does not depend at all on the trajectory, bounded and chaotic or regular and unbounded. The only thing that is common to classical and quantum energy is the inverse time behaviour $\left(a^{-1}(t)\right)$ for $t \rightarrow 0$.

Finally we discuss the boundedness of E . Every solution of (3.1) depends on three parameters $\mathrm{m}, \xi$, and the spectral variable $\delta(2-\delta)$. There is a restriction on $\mathrm{m}$ and $\xi$, $\mathrm{K}^{2}(\mathrm{~m}, \xi)$ in eq. (3.7) must be positive in order to fulfil the boundary conditions. The requirement on $\mathrm{E}$ of uniform boundedness from below, simultaneously for $\mathrm{t}$ and $\delta(2-\delta)$ 
for fixed $\mathrm{m}, \xi$, imposes further restrictions on the spectral variable $\delta(2-\delta)$. At first we note that in eq. (3.13) we have to require $1-6 \xi>0$. If this is not satisfied we exclude the solution corresponding to the discrete eigenvalue, as it is the case for $m=0$. The smallest value that the second term in (3.14) periodically admits is - $|\mathrm{B}|$. The necessary and sufficient condition insuring boundedness of $E$ from below for $t \rightarrow 0$ is thus $\mathrm{A}-|\mathrm{B}|>0$, or

$$
\begin{aligned}
& \mathrm{F}(\tilde{v}):=\tilde{v}^{2}+1-6 \xi-\sqrt{\tilde{v}^{2}+1}|1-6 \xi| \frac{1}{\cosh (\pi \kappa)} \sqrt{1+\frac{\sinh ^{2}(\pi \kappa)}{\cosh ^{2}(\pi \tilde{v})}}>0 \\
& 0<\tilde{v}<\infty, \tilde{v}=\sqrt{\delta(2-\delta)-1} \text {. Note that } F(\tilde{v})=1-6 \xi-|1-6 \xi|+O\left(\tilde{v}^{2}\right) .
\end{aligned}
$$

There is for given $\mathrm{m}, \xi$ at most a finite interval to be excluded from the spectrum of $\Delta_{B^{3}}$ to ensure $E(v, t)>$ const. $(m, \xi)$ for $t \rightarrow 0$. From the asymptotic formulae ( 3.11, 3.12 ) and ( 3.15$)$ and from the asymptotic behaviour of the exact solution [8] for $\tilde{v} \approx$ const.exp $(\Lambda t) \rightarrow \infty,|\varphi(\tilde{v}, t)| \approx$ const.exp $(-3 / 2 \Lambda t)$, which gives a lower bound for the second term in (3.6) ) we conclude that the energy functional is bounded from below, uniformly for all $t, v$ with the exception of the excluded interval. It can be made positive definite by adding a finite constant, independent of $v$ and $t$.

\section{Conclusion and outlook}

The global topological structure of the universe reveals interesting relations between the classical and quantal formulation of dynamics. The multiple connectivity of the spacelike slices produces on the one hand chaotic trajectories, on the other hand bound states, square-integrable chaotic wave fields, both determined by the limit sets of the covering groups of the slices. In Section III we discussed in some detail in the context of de Sitter space the asymptotic time behaviour of the energy of such wave fields, and its dependence on the Hausdorff dimension of the limit sets.

We believe that the decisive influence of topology will survive if we relax the restriction of constant curvature, imposed on the spacelike slices. In fact, local deviations from homogeneity and isotropy and even singularities can be described by perturbations of the Poincaré metric (by adding to it a symmetric tensor field automorphic with respect to the discrete group $\Gamma$, or by an automorphic conformal factor), and the described picture should not change. For the practical construction of such perturbations in terms of Poincaré series see [9]. The covering space is then still $\mathrm{B}^{3}$, the covering group $\Gamma$, the 
polyhedral tiling remains the same, though the polyhedral faces are not any more geodesic planes. The lifts of the chaotic geodesics in the perturbed metric emanate again from the same limit set, and the bound state wave functions should still have an integral representation in terms of weighted automorphic forms, supported on the limit set.

Finally, the classical electromagnetic field should be treatable in similar terms, each of its components satisfying a Klein-Gordon equation, one has then to cope with potential terms of the form $\hat{R}_{\mu}{ }^{v} A_{v}$ and the gauge fixing terms that lead to a coupling among its components.

\section{Acknowledgement}

The author acknowledges the partial support of the European Communities in their science programme under grant $\mathrm{B} / \mathrm{SC} 1 *-915078$.

\section{References}

[1] Ahlfors L.V. (1981) Möbius Transformations in Several Dimensions, Lecture Notes (Univ. Minnesota).

[2] Bers L. (1970) On Boundaries of Teichmüller Spaces and on Kleinian Groups, Ann. Math. 21, 570-600.

[3] Hejhal D.A. (1987) Boundary-Groups, Degenerating Riemann Surfaces, and Spectral Theory, Univ. of Minnesota preprint.

[4] Landau L.D. and E.M.Lifshitz (1971) The Classical Theory of Fields, Pergamon, London.

[5] Misner C.W., K.S. Thorne and J.A.Wheeler ( 1973) Gravitation, Freeman, New York.

[6] Patterson S.J. (1987) Lectures on Measures on Limit Sets of Kleinian Groups, in : Analytical and Geometrical Aspects of Hyperbolic Space, London, Math. Soc. Lecture Notes 111, 281-323; D.B.A. Epstein, ed.(Cambridge Univ. Press, London).

[7] Tomaschitz R. (1989) On the Calculation of Quantum Mechanical Ground States from Classical Geodesic Motion on Certain Spaces of Constant Negative Curvature, Physica D 34, 42-89.

[8] Tomaschitz R. (1991) Relativistic Quantum Chaos in Robertson-Walker Cosmologies, J. Math. Phys. 32, nr.10 (to appear).

[9] Tomaschitz R., Chaotic Dynamics in General Relativity, to be published in the Proceedings of a Nato Advanced Research Workshop on Chaotic Dynamics, held July 11-20 1991 in Patras, Greece; T. Bountis, ed. (Plenum, NY). 